\title{
Brugada Syndrome: A Review
}

\author{
Laxmi Narayan Goit ${ }^{*}$, Shaning Yang² \\ ${ }^{1}$ Department of Cardiology, The First Affiliated Hospital of Yangtze University, Jingzhou, China \\ ${ }^{2}$ Department of Cardiology, Clinical College of Yangtze University and The First Affiliated Hospital to Yangtze University, \\ Jingzhou, China \\ Email: *laxmi_goit@hotmail.com
}

How to cite this paper: Goit, L.N. and Yang, S.N. (2019) Brugada Syndrome: A Review. Yangtze Medicine, 3, 235-252. https://doi.org/10.4236/ym.2019.34023

Received: November 5, 2018

Accepted: June 27, 2019

Published: June 30, 2019

Copyright $\odot 2019$ by author(s) and Scientific Research Publishing Inc. This work is licensed under the Creative Commons Attribution International License (CC BY 4.0).

http://creativecommons.org/licenses/by/4.0/

\begin{abstract}
The Brugada syndrome is a form of cardiac arrhythmia, characterized by electrocardiographic ST-Segment elevation in right precordial leads that affect young male patient, predisposing to malignant ventricular arrhythmia and sudden cardiac deaths. The majority of the patients with Brugada syndrome remain asymptomatic, however, patient can present with symptom like syncope, palpitation and aborted sudden cardiac death. Several pathogenic genes have been identified as associated with the disease but SCN5A is most prevalent one. The Brugada syndrome is diagnosed by typically cove shaped ST-segment elevation of $>2 \mathrm{~mm}$ in greater than one precordial lead V1 and V2, occurring spontaneously or after provocative drugs test with IV administration of class 1 antiarrhythmic drug such flecainide or Ajmaline. Risk stratification and the need for treatment depend on the patient symptom, electrocardiography, family history and electrophysiological study. The treatment by implantable cardioverter defibrillators, the only effective treatment to date is appropriate. Other treatment options included pharmacological therapy (Quinidine) and Radiofrequency ablation of ventricular ectopies. This brief review focuses on epidemiology of Brugada syndrome, Genetic basis, mechanism, clinical presentation, ECG changes, risk stratification, Diagnostic criteria and management.
\end{abstract}

\section{Keywords}

Sudden Cardiac Death, Cardiac Arrhythmia, Ventricular Fibrillation, Risk Stratification, Implantable Cardioverter Defibrillator

\section{Introduction}

Brugada syndrome was initially described by Pedro and Joseph Brugada in 1992 [1]. Brugada syndrome is a rare cardiac arrhythmia characterized by electrocar- 
diographic right bundle branch block and persistent ST-Segment elevation in right precordial lead. Brugada syndrome is defined electrocardiographically by a characteristic patter including J-Point and ST-segment elevation of $2 \mathrm{~mm}$ or greater, followed by a negative $\mathrm{T}$ wave in the right precordial leads [1]. It is closely linked to SCN5A gene mutation affecting the sodium channel function [1]. The typical arrhythmia of Brugada syndrome is polymorphic ventricular tachycardia which can potentially degenerate to ventricular fibrillation and high risk of sudden cardiac death, predominantly in younger male patient with structural normal heart [1]. Repetitive monomorphic ventricular tachycardia including ventricular flutter occurring in Brugada syndrome is potentially lethal events. Most patients with Brugada syndrome are diagnosed only after cardiac arrest [2]. The symptom usually appears around 40 years of age and males are more often symptomatic than females probably from the influence of hormones and gender distribution of ion channels across the heart. Currently, the only proven effective strategy for preventing sudden cardiac death (SCD) in Brugada syndrome patient is the use of implantable cardioverter defibrillators.

\section{Epidemiology}

Brugada syndrome has been estimated to account for $20 \%$ of sudden cardiac death in the absence of structural heart disease and may be responsible for between $4 \%$ and $12 \%$ of all patients with sudden cardiac death [3]. The prevalence of Brugada syndrome also varies greatly according to location, being higher in east and south-east Asian population (in Thailand, Japan and Philippines) than of European descent [4] [5] [6]. Current prevalence rates estimates of 5/10,000. In North America and Western Europe the prevalence of the syndrome is relatively lower [7]. A Japanese study reported the prevalence of a type-1 ECG pattern to be $12 / 10,000$ [4]. In the endemic areas the Brugada syndrome may represent, after excluding accidents, the leading cause of death in individual younger than 40 years [8].

\section{Genetics}

Brugada syndrome is hereditary disease with an autosomal dominant patter of transmission and variable penetrance [9]. However, up to $60 \%$ may be sporadic that is absent in other relatives [10]. In 1998, the first pathogenic mutation in the SCN5A gene was identified, which encodes for the alpha-subunit of the cardiac sodium channel [11]. Since them, more than 350 pathogenic mutations in several genes have been published (SCN5A, GPD1L, SCN1B, SCN2B, SCN3B, RANGRF, SLMAP, KCNE3, KCNJ8, HCN4, KCNE5, KCND3, CACNA1C, CACNB2B, CACNA2D1 and TRPM4) [12]. These genes encode subunit of cardiac sodium, potassium, and calcium channel as well as genes involved in regulation of channels. Besides SCN5A genes, many BS related genes play role in regulating sodium channel function. It has been suggested that the gain of function caused by a mutation in the genes encoding channels that conduct outward 
potassium currents (KCND3, KCNE3, KCNE5, and KCNJ8) predispose affected individuals to develop Brugada syndrome phenotype [13]. Brugada syndrome susceptibility genes were also found among calcium channels (CACNA1C, CACNB2b and CACNA2D1). The loss of function mutations involving the L-type calcium channel subunits, encoded by the CACNA1C, CACNB2B and CACNA2D1 genes respectively, in probands with Brugada syndromes. These genes only account for small percentage of Brugada syndrome [14]. Despite the high number of gene mutation, only about $35 \%$ of Brugada syndrome patient have been determined to have a genetic causes. Of them, nearly $30 \%$ carry pathogenic mutation in the SCN5A gene [15]. All other genes together are responsible for about $5 \%$ of all Brugada syndrome cases. Therefore $65 \%$ of cases do not have genetic origin. Several factors could explain the high number of Brugada syndrome patient without genetic alteration after genetic screening. The pathogenic mutation associated with Brugada syndrome could be localized in unknown genes or disease could be related to epigenetic factors, mainly DNA methylation, post translational modification and RNA Mechanism [16] [17].

\section{Mechanism}

Brugada syndrome was initially thought of as a channelopathy, an exclusively electrophysiological disorder produced by dysfunction of a cardiac ion channel involved in generation of the action potential in structural normal heart [1] [18] [19] [20]. More study reported patient with the Brugada ECG patterns showing structural abnormalities of right ventricle [21] [22]. This supports the theory that the pathophysiological mechanism of Brugada syndrome may be due to slowing of conduction in the right ventricle, accompanied by mild structural abnormalities [23]. Currently no single causal factor link all patient and thus the pathophysiological mechanism remain elusive [23]. Two hypotheses have been proposed-depolarisation theory and repolarisation theory, to explain the pathophysiology of type-1 ECG pattern and susceptibility to ventricular arrhythmia [20] [23] [24].

\subsection{Repolarisation Hypothesis}

According to this hypothesis, rebalancing of current at the end of phase 1 of the action potential (AP) leads to accentuation of the AP notch in the epicardium of the right ventricle. Such accentuation was believed responsible for the characteristic ST-Segment elevation [1] [18] [19] which is shown in Figure 1.

In the presence of pathophysiological condition such as a SCN5A mutation (leading to loss of function of sodium channel), outward current overwhelm inward current and the epicardium may then exhibit all-or-none repolarisation at the point where the phase- 1 action potential reaches $-30 \mathrm{mv}$. The resulting loss of action potential dome at some but not all, epicardial sites could create marked epicardial dispersion of repolarisation. This gives rise to a transmural voltage gradient leading to transmural dispersion of repolarisation between the 


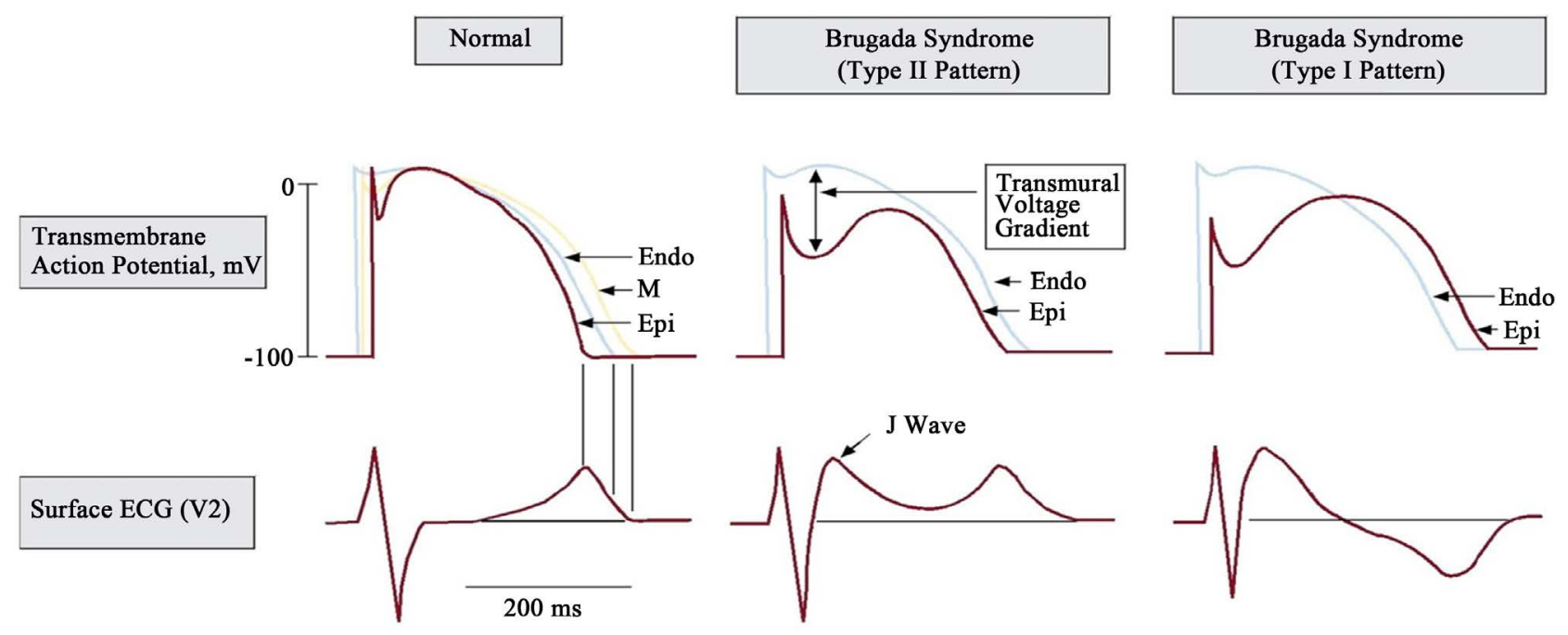

Figure 1. Schematic representation of the repolarisation disorder hypothesis [25]. (Reproduced with permission). Endo-Endocardium, M-Myocardium, EPI-Epicardium.

epicardium and endocardium which manifests as the characteristic ST-Segment elevation of the ECG [20] [21]. The T-wave remains positive when epicardial repolarisation precedes that of myocardial and endocardial regions resulting in characteristic type-2 ECG [25]. When sites in the epicardial region exhibit prolongation of action potential, reversed direction of repolarisation across the right ventricle wall may ensue, producing the inverted T-wave and resulting in the type-3 coved type ECG [25]. The repolarisation hypothesis could also explain the mechanism of ventricular arrhythmia initiation. When epicardial dispersion of repolarisation and transmural dispersion of repolarisation occur via-or-none repolarisation at some epicardial site, an arrhythmogenic substrate is formed. This creates a vulnerable window when an action potential can propagate from sites with normal repolarisation to sites with early repolarisation. The conduction of an action potential dome lead to local re-excitation via a phase- 2 re-entry mechanism at the sites of early repolarisation and facilitates the development of extrasystoles beats from these sites [20] [25] [26]. This extrasystoles beats have been shown to initiates circus movement re-entry [26] and thus give rise to malignant ventricular arrhythmia [20].

\subsection{Depolarisation Hypothesis}

The depolarisation hypothesis is for the Brugada ECG and susceptibility to ventricular arrhythmia propose a conduction delay in the right ventricular outflow tract [23] [27]. The action potential is not fundamentally altered; rather conduction delay in the right ventricular outflow tract slows action potential development in right ventricle. This pathophysiological mechanism was believed to result in Brugada ECG pattern and provide an arrhythmogenic substrate via a re-entry circuit.

A third hypothesis involves a combination of both depolarization and repolarization abnormalities, as our groups has found using Frank vectorcardiograms 
to compare patient with Brugada syndrome vs. patient with complete and incomplete right bundle branch block [28].

The Brugada syndrome is recognized as disorders with no structural defects [9], there is some evidence for involvement of mild structural abnormalities [29], which were not previously detectable by conventional cardiac imaging [1] [30]. Structural abnormalities found in the Brugada syndrome patient include focal fibrosis, myocarditis, apoptosis and fibrofatty replacement of the right ventricle free wall with right ventricle enlargement, dilation and right ventricle outflow tract enlargement [29] [30] [31]. It is suggested that such structural abnormalities play role in Brugada syndrome [29] [31] by contributing to slower conduction [32] [33].

\section{Clinical Presentation of Brugada Syndrome}

The clinical presentation can range from no symptom that is asymptomatic to sudden cardiac death. Initial presenting symptoms include palpitation, syncope, seizure and nocturnal agonal respiration [34]. About 25\% of patients suffering from sudden cardiac death had already experienced a syncope episode due to arrhythmic complications such as polymorphic ventricular tachycardia or ventricular fibrillation [35]. In up to $20 \%$ of cases Supraventricular arrhythmia may exist, mainly atrial fibrillation [36]. Although Atrioventricular nodal reentry tachycardia and Wolff-Parkinson white syndrome has also been reported [37] [38]. Additionally an association with sinus dysfunction has been described [39]. Symptom occur more frequently during rest or during sleep (especially between 12 am and 6 am and rarely occurs during day time), febrile episodes or under predominance of vagal activity [40] [41] [42]. It is rare that they occur during exercise. The variation of the sympathetic-parasympathetic balance, hormonal and other metabolic factors are likely to contribute to this pattern. Increased vagal tone decrease acetylcholine mediated calcium current, which could lead to arrhythmogenesis by phase- 2 reentry [43]. The causes of death in Brugada syndrome is ventricular fibrillation.

\section{Electrocardiogram (ECG) Changes in Brugada Syndrome}

The diagnosis of Brugada syndrome is based on both clinical finding and characteristic ECG pattern that occurs spontaneously or are induced by the use of sodium channel blocking agents. There are three known ECG subtype that can be detected in more than one of the right precordial leads (V1-V3) and have a diagnostic values [44].

\subsection{Type-1 ECG Pattern}

The type-1 ECG pattern is characterized by a Coved shape ST-Segment elevation greater than $2 \mathrm{~mm}(0.2 \mathrm{mv})$ followed by an inverted T-Wave in one or more of the right precordial lead, which occurs with or without provocation by sodium channel blocking agent [9] as shown in Figure 2. 


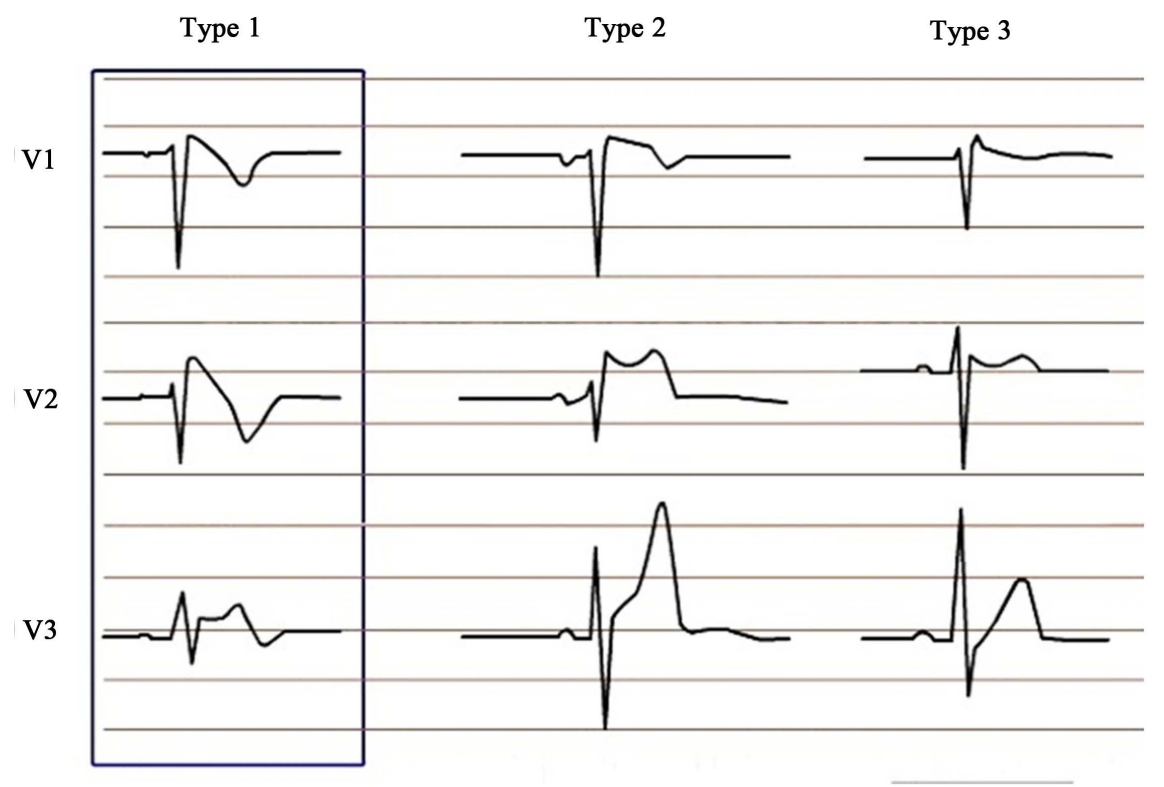

Figure 2. Figure showing pattern of ECG in Brugada Syndrome. Type-1 ECG pattern shows Coved ST-Segment elevation $>2 \mathrm{~mm}$ is followed by a negative T-Wave, with little or no isoelectric line separation that feature is present in from V1 to V2 in right precordial leads. Type-2 ECG patterns also characterized by ST-Segment Elevation followed by positive or biphasic T-wave that result in saddle back configuration. Type-3 ECG patterns show right precordial ST-Segment elevation $<1 \mathrm{~mm}$ with saddle morphology [9].

This pattern must ensue alongside one of the following history of ventricular fibrillation, polymorphic ventricular tachycardia, and family history of unexplained sudden cardiac death in person older than 45 years, family history of coved-type ECG, inducibility of ventricular tachycardia with programmed electrical stimulation, syncope episodes or nocturnal agonal Respiration [9].

\subsection{Type-2 ECG Pattern}

In the type-2 ECG pattern, the ST-Segment resembles a saddleback, with an ST-Segment elevation at least $2 \mathrm{~mm}(0.2 \mathrm{mv})$, a trough of the ST-Segment elevation of at least $1 \mathrm{~mm}$ and then either a positive or biphasic T-Wave [9], shown in Figure 2.

\subsection{Type-3 ECG Pattern}

In the type-3 ECG pattern consists of either a Coved (type-1 like) or saddleback (type-2 like) ST-Segment elevation between 1 and $2 \mathrm{~mm}$. Both type- 2 and type- 3 ECG finding occurs spontaneously without use of sodium channel blocking agent but they themselves they are not considered diagnostic, as shown in Figure 2.

If the type- 2 or type-3 ECG pattern converted to type-1 ECG pattern after provocation by sodium channel blocking agents and at least one of the above mentioned characteristic finding is present, a diagnosis of Brugada syndrome is considered. 


\subsection{Other ECG Finding in Brugada Syndromes}

The PR interval is often increased $>200 \mathrm{~ms}$. There is a P-wave abnormality that is biphasic $\mathrm{P}$ wave and QRS complex is widening and fragmented QRS [45]. The prolongation of QT intervals in right precordial leads is also seen in Brugada syndrome [46].

\section{Risk Stratification}

Risk stratification of Brugada syndrome is aimed to identifying individuals most liable to sudden cardiac death, so that they can receive appropriate management. It is well accepted that etiology of Brugada syndrome is multifactorial, involving genetic, environmental and hormonal components that contribute to phenotype manifestation. Symptomatic presentations included aborted sudden cardiac death, syncope, seizure and nocturnal agonal respiration. Asymptomatic individuals should be assessed for relevant family history of sudden cardiac death. Individual with diagnosis of Brugada syndrome based on spontaneous or induced type-1 ECG pattern may present with or without symptom.

The Brugada syndrome typically manifests in adulthood, with mean age of 41 \pm 15 years [9]. However the Brugada syndrome may manifest in children and the elderly, patients have been diagnosed at 2 days of life to 80 years [9]. It is $8-10$ times more prevalent in men than in women [9] [35] [47] [48] [49]. This could be due to constitutional differences in transmembrane ionic current between two sexes [50]. The evidences suggests hormonal influence on the phenotypic gender differences, such as type-1 ECG regression after castration in men with prostate neoplasia [51] and presence of high concentration of testosterone in men with Brugada syndrome than control [52]. The family history is present in about $20 \%-30 \%$ of the patients [53]. The prognosis also differ with gender, with a 4.5 - 5.5 times higher risk of SCD in men than women [48] [54].

The international Registry of Brugada syndrome reported that $25 \%$ of this population experienced sudden cardiac death or ventricular fibrillation during life time at mean age of 42 years [54]. Recently, in a large series of patients, aborted sudden cardiac death frequency was estimated to be $7.7 \%, 1.9 \%$ in patient with syncope only and $0.5 \%$ in entirely asymptomatic [55]. Other factors denoting increased risk of an adverse cardiac event included aborted sudden cardiac death, syncope spontaneous or unmask type-1 ECG pattern, family history of sudden cardiac death and inducibility of ventricular tachycardia or ventricular fibrillation during an electrophysiological study. Among patients presenting with aborted sudden cardiac death, the risk of its recurrence or ventricular fibrillation was $62 \%$ with a mean follow up time of 54 month. These patients have highest risk of future cardiac event so these patients have the most compelling indication for treatment with an implantable cardioverter defibrillator (ICD), the only treatment option demonstrated to be effective to data. Even if there is no history of cardiac arrest, Brugada syndrome is reported to carry an $8.2 \%$ risk of sudden cardiac death or ventricular fibrillation over 25 month [48]. In patient 
with Brugada syndrome, inducibility of ventricular arrhythmia by electrophysiological means is predictors of poorer prognostic outcome. Inducible ventricular tachycardia or ventricular fibrillation by electrophysiological study warrants ICD treatment even for asymptomatic patient if they show a spontaneous type-1 ECG pattern or family history of sudden cardiac death [54]. All other patient warrant close follows up.

\section{Diagnostic Criteria of Brugada Syndrome}

\section{Consensus statement regarding making diagnosis of Brugada syndrome:}

The first consensus report of 2002 Proposed using ECG criteria alone and three Subtypes (type-1 pattern, type-2 pattern and type-3) of ECG feature have been recognized [56].

The second consensus published in 2005 for the diagnosis of Brugada syndrome the following are the necessary [9].

1) Presence of type-1 ECG pattern-descends ST-Segment elevation $\geq 2 \mathrm{~mm}$ in more than one right precordial lead, spontaneously or after exposure to sodium channel blocking drugs.

2) The presence of one of the following clinical manifestation:

- Documented ventricular fibrillation, polymorphic ventricular tachycardia.

- Family history of sudden cardiac death at $<45$ years of age without acute coronary syndrome.

- Coved shaped ECG in family members.

- Inducibility of ventricular tachycardia with programmed stimulation.

- Clinical history of non-vasovagal syncope or nocturnal agonal respiration.

In 2005, there have several clinical studies on sensitivity and specificity of ECG diagnosis of Brugada syndrome. Therefore proposed amendments to the diagnostic criteria [57], the Brugada syndrome is diagnosed when type-1 ECG pattern ST-Segment elevation is observed either spontaneously or after intravenous administration of sodium channel blockers in at least one right precordial lead (V1 and V2), placed in a standard or superior position.

The latest consensus statement of 2013 does not mentioned these additional clinical feature and only mentions the ECG features [50].

The Expert consensus statement, diagnosis and management of patients with inherited primary arrhythmic syndrome 2013 [58]:

1) Brugada syndrome is diagnosed in patient with ST-Segment elevation with type-1 morphology $\geq 2 \mathrm{~mm}$ in more than more lead among the right precordial lead V1, V2 positioned in second, third or fourth intercostals space occurring spontaneously or after provocative drugs test with intravenous administration of class 1 antiarrhythmic drugs.

2) Brugada syndrome is diagnosed in patients with type- 2 or type-3 ST-Segment elevation in more than one lead among the right precordial lead V1, V2 positioned in the second, third or fourth intercostals space when provocation drug test with intravenous administration of class 1 antiarrhythmic drugs in- 
duces type-1 ECG morphology.

\section{Pharmacological test: Electrocardiogram unmasking.}

When there is a clinical suspicion of Brugada syndrome without presenting an ECG with spontaneous type-1 ECG pattern, patient should undergo a pharmacological challenges test with sodium channel blocking drugs (Ajmaline, Flecainide, Procainamide, or Pilsicainide) [59], Table 1 shows the drug used, dose and routes of administration. Other pharmacological agent also induce Brugada like ECG pattern include calcium channel blockers, beta blockers, antianginal drugs, psychotic drugs, alcohol, cocaine and bupivacaine [59].

The test is performed under continuous Electrocardiographic monitoring. At the beginning and end of the drugs test leads V1 and V2 should placed in the $2^{\text {nd }}$ and $3^{\text {rd }}$ intercostals space, as it increase the sensitivity of the ECG for detecting the diagnostic patterns [60] [61]. The test is considered positive only if a type-1 ECG pattern is obtained, shown in Figure 3 and should be discontinued in the early onset of frequent ventricular premature beat or others ventricular arrhythmia [9]. Data available to date suggest that Ajmaline is the most effective in the diagnosis of Brugada syndromes [62].

\section{Modulating Factors and Differential Diagnosis}

Exposure to certain drugs or ionic change can produce ST-Segment elevation suggestive of Brugada syndrome, which may represent a certain genetic predisposition. Fever also modulates the phenotype and the risk of arrhythmias by

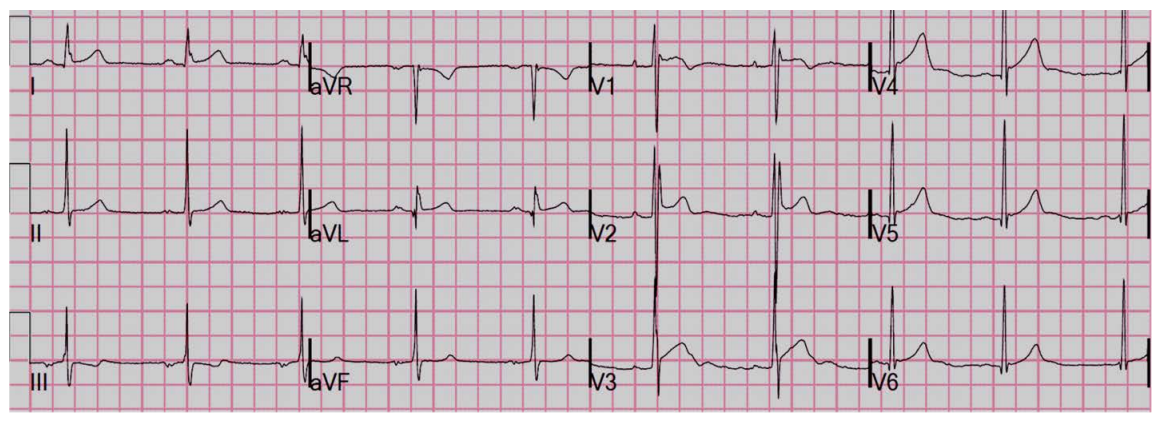

Figure 3. 12 leads Electrocardiogram (ECG) showing Brugada syndrome type-1 ECG pattern showed coved ST-Segment elevation $>2 \mathrm{~mm}$ is followed by negative T-Wave with little or no isoelectric separation in right precordial leads from V1 to V3 [9].

Table 1. Sodium channel blocking agents that can be used in provocation test to unmask the ECG in Brugada Syndrome [59].

\begin{tabular}{clcc}
\hline & Drugs & Doses & Routes of Drugs Administration \\
\hline - & Ajmaline & $1 \mathrm{mg} / \mathrm{kg}$ over 5 minute & Intravenous \\
- & Flecainide & $2 \mathrm{mg} / \mathrm{kg}$ over 10 minute & Intravenous \\
- & Procainamide & $10 \mathrm{mg} / \mathrm{kg}$ over 10 minute & Intravenous \\
- & Pilsicainide & $1 \mathrm{mg} / \mathrm{kg}$ over 10 minute & Intravenous \\
- & Flecainide & $400 \mathrm{mg}$ & Oral \\
\hline
\end{tabular}


accentuating the sodium channel inactivating, unmasking the type 1 pattern and triggering ventricular arrhythmias [63] [64] [65]. If any of these factors is present, it must be corrected. It is also important to exclude other causes of ST-Segment elevation before making the diagnosis. Some other causes of ST-Segment elevation are shown in Table 2.

\section{Management of Brugada Syndrome}

The treatment options for Brugada syndrome have been related to device related and. Pharmacological therapies. However education and prevention of arrhythmias via lifestyle awareness should also be considered.

\subsection{Lifestyle Measures}

The following life style measures are recommended in patients with diagnosis of Brugada syndrome:

1) Avoidance of drugs that aggravates or induces ST-Segment elevation in right precordial leads [68].

2) Avoidance of excessive alcohol intake.

3) Immediate treatment of fevers with antipyretic drugs.

Management of acute malignant arrhythmias or electrical storms included early defibrillators and resuscitation of patient followed by admission to specialized cardiac care unit. Any provocative factors such as fever should be treated with antipyretics and cold sponging. Any intake of arrhythmogenic drugs should be discontinued. Pharmacological treatment with isoprenaline $1-2 \mu \mathrm{g}$ bolus intravenously followed by continuous infusion of $0.15-0.2 \mu \mathrm{g} /$ minutes or Quinidine 300 - $1500 \mathrm{mg} /$ day has been used for electrical stroma.

\subsection{Device Management}

The currently, the only proven effective strategy for preventing sudden cardiac death in Brugada syndrome patients is the use of an implantable cardioverter defibrillators (ICD) [54]. The most recently consensus published in 2013, report that implantable cardioverter defibrillators is the only established form of effective treatment to all symptomatic patients presenting with Brugada syndrome [57]. All asymptomatic patients should undergo further risk stratification and

Table 2. Differential diagnosis of Brugada syndrome [66] [67].

\begin{tabular}{ll}
\hline Following are the differential diagnosis of Brugada syndrome: \\
\hline - & Atypical right bundle branch block \\
- & Left ventricular hypertrophy \\
- & Acute pericarditis/myocarditis \\
- & Acute pulmonary thromboembolism \\
- & Aortic dissecant aneurysm \\
- & Arrhythmogenic right ventricular dysplasia
\end{tabular}


electrophysiological study to accesses their indication for an ICD [57]. ICD is indicated in patient with a spontaneous diagnostic type-1 ECG patter, who have a history of syncope juged to be likely caused by ventricular arrhythmias and also considered in patients with a diagnosis of Brugada syndrome who develop ventricular fibrillation during programmed electrical stimulation.

In asymptomatic patients whose type-1 ECG patterns is documented only after administration of sodium channel blockers, it is not recommended to performs regular monitoring without electrophysiological study for risk stratification nor ICD implantation [57]. Drugs that can causes Brugada like change in ECG are avoided and included: class 1 antiarrhythmic drug like flecainide, beta blockers, and Tricyclic antidepressant drugs, phenothiazines, Fluoxetine, anesthetics agent such as bupivacaine, procaine and alcohol [68] [69].

Complication of implantable Cardioverter defibrillator (ICD):

Although ICD are very effective in treating ventricular fibrillation and implantation procedure may lead to some surgical complication like bleeding, pneumothorax, perforation of vascular structures, cardiac tamponade and infection. Post implantation issues included displacement or fracture of leads and need to replace the device because of battery depletion.

\subsection{Pharmacological Therapy}

It has been shown that Quinidine, antiarrhythmic with blocking activity in the Ito and Ikr currents, reduces the incidence of arrhythmias induced in patient with Brugada syndrome [70] and has been used successfully in certain clinical conditions, such as the treatment of arrhythmia storm [71], multiple shock [72] [73] or alternative to ICD in children at risk of arrhythmia [74]. However Quinidine often causes side effect like diarrhoea, thrombocytopenia and hepatitis that resolve after drug discontinuation. The main concern relates to the potential pro-arrhythmic activity of Quinidine, there is risk of torsades de point, so there is a close monitoring is required during the first few days of therapy.

Quinidine therapy may be alternative to ICD, who are ineligible or person who refused such an intervention.

\subsection{Catheter Ablation}

After finding that ventricular fibrillation events in Brugada syndrome patient is caused by ventricular premature beats, catheter ablation of ventricular ectopies, was proposed as a therapeutic measure [75]. Thus, in 2011, Nademanee et al. published the first study showing preventions of ventricular fibrillation in Brugada syndrome patients by catheter ablation over the anterior right ventricular outflow tract epicardium [76].

\section{Conclusion}

The Brugada syndrome is an inherited syndrome characterized by typically ST-segment elevation in right precordial lead that affects young individual, 
mostly male, predisposing to ventricular arrhythmia and sudden cardiac death. It is diagnosed by typically cove shaped ST-Segment elevation of $>2 \mathrm{~mm}$ and negative T-wave with little or no isoelectric separation in more than one in right precordial leads. Several genetic mutations of the sodium, calcium and potassium channel have been involved. The majority of the patients with BS remain asymptomatic, however, the most frequent symptom is syncope or SCD secondary to PVT or VF. Risk stratification is mainly based on symptom and surface electrocardiogram. Implantable cardioverter defibrillator is the only proven effective strategy for preventing SCD in BS patients. However, there are still dought and controversies regarding the underlying mechanism, the influences of different modulating factors and how to stratify the risk and treatment asymptomatic patients. The patient diagnosed with Brugada syndrome is counseled, such that they and their relatives are informed.

\section{Acknowledgements}

This review article is supported by the National Natural Science Foundation of China (31700736), Hubei Province Natural Science Foundation of China (2016CFB180), Hubei Province Health and Family Planning Scientific Research Project (WJ2016Y07), Hubei Province Scientific and Technological Research Project (Q20171306), Jingzhou Science and Technology Development Planning Project (JZKJ15063) and the Yangtze Fund for Youth Teams of Science and Technology Innovation (2016CQT04).

\section{Conflicts of Interest}

There authors have no Conflicts of interest to declare.

\section{References}

[1] Brugada, P. and Brugada, J. (1992) Right Bundle Branch Block, Persistent ST Segment Elevation and Sudden Cardiac Death: A Distinct Clinical and Electrocardiographic Syndrome. A Multicenter Report. Journal of the American College of Cardiology, 20, 1391-1396. https://doi.org/10.1016/0735-1097(92)90253-I

[2] Paul, M., et al. (2007) Role of Programmed Ventricular Stimulation in Patients with Brugada Syndrome: A Meta-Analysis of worldwide Published Data. European Heart Journal, 28, 2126-2133. https://doi.org/10.1093/eurheartj/ehm116

[3] Benito, B., Brugada, J., Brugada, R. and Brugada, P. (2009) Brugada Syndrome. Revista Española de Cardiología, 62, 1297-1315. https://doi.org/10.1016/S0300-8932(09)73082-9

[4] Miyasaka, Y., et al. (2001) Prevalence and Mortality of the Brugada-Type Electrocardiogram in One City in Japan. Journal of the American College of Cardiology, 38, 771-774. https://doi.org/10.1016/S0735-1097(01)01419-X

[5] Greer, R.W. and Glancy, D.L. (2003) Prevalence of the Brugada Electrocardiographic Pattern at the Medical Center of Louisiana in New Orleans. Journal of the Louisiana State Medical Society, 155, 242-246.

[6] Hermida, J.S., Lemoine, J.-L., Aoun, F.B., Jarry, G., Jean-Luc, R. and Quiret, J.-C. (2000) Prevalence of the Brugada Syndrome in an Apparently Healthy Population. 
American Journal of Cardiology, 86, 91-94.

https://doi.org/10.1016/S0002-9149(00)00835-3

[7] Gallagher, M.M., et al. (2008) Prevalence and Significance of Brugada-Type ECG in 12,012 Apparently Healthy European Subjects. International Journal of Cardiology, 130, 44-48. https://doi.org/10.1016/j.ijcard.2007.07.159

[8] Nademanee, K. (1997) Sudden Unexplained Death Syndrome in Southeast Asia. American Journal of Cardiology, 79, 10-11. https://doi.org/10.1016/S0002-9149(97)00113-6

[9] Antzelevitch, C., et al. (2005) Brugada Syndrome: Report of the Second Consensus Conference: Endorsed by the Heart Rhythm Society and the European Heart Rhythm Association. Circulation, 111, 659-670. https://doi.org/10.1161/01.CIR.0000152479.54298.51

[10] Schulze-Bahr, E., et al. (2003) Sodium Channel Gene (SCN5A) Mutations in 44 Index Patients with Brugada Syndrome: Different Incidences in Familial and Sporadic Disease. Human Mutation, 21, 651-652. https://doi.org/10.1002/humu.9144

[11] Chen, Q., et al. (1998) Genetic Basis and Molecular Mechanism for Idiopathic Ventricular Fibrillation. Nature, 392, 293-296. https://doi.org/10.1038/32675

[12] Nielsen, M.W., Holst, A.G., Olesen, S.-P. and Olesen, M.S. (2013) The Genetic Component of Brugada Syndrome. Frontiers in Physiology, 4, 179.

https://doi.org/10.3389/fphys.2013.00179

[13] Delpon, E., et al. (2008) Functional Effects of KCNE3 Mutation and Its Role in the Development of Brugada Syndrome. Circulation: Arrhythmia and Electrophysiology, 1, 209-218. https://doi.org/10.1161/CIRCEP.107.748103

[14] Antzelevitch, C., et al. (2007) Loss-of-Function Mutations in the Cardiac Calcium Channel Underlie a New Clinical Entity Characterized by ST-Segment Elevation, Short QT Intervals, and Sudden Cardiac Death. Circulation, 115, 442-449. https://doi.org/10.1161/CIRCULATIONAHA.106.668392

[15] Kapplinger, J.D., et al. (2010) An International Compendium of Mutations in the SCN5A-Encoded Cardiac Sodium Channel in Patients Referred for Brugada Syndrome Genetic Testing. Heart Rhythm, 7, 33-46.

https://doi.org/10.1016/j.hrthm.2009.09.069

[16] Webster, A.L., Yan, M.S. and Marsden, P.A. (2013) Epigenetics and Cardiovascular Disease. Canadian Journal of Cardiology, 29, 46-57. https://doi.org/10.1016/j.cjca.2012.10.023

[17] Kim, G.H., Ryan, J.J. and Archer, S.L. (2013) The Role of Redox Signaling in Epigenetics and Cardiovascular Disease. Antioxidants \& Redox Signaling, 18, 1920-1936. https://doi.org/10.1089/ars.2012.4926

[18] Link, M.S., et al. (2001) Clinical Cardiac Electrophysiology Fellowship Teaching Objectives for the New Millennium. Journal of Cardiovascular Electrophysiology, 12, 1433-1443. https://doi.org/10.1046/j.1540-8167.2001.01433.x

[19] Yan, G.X. and Antzelevitch, C. (1996) Cellular Basis for the Electrocardiographic J Wave. Circulation, 93, 372-379. https://doi.org/10.1161/01.CIR.93.2.372

[20] Yan, G.X. and Antzelevitch, C. (1999) Cellular Basis for the Brugada Syndrome and Other Mechanisms of Arrhythmogenesis Associated with ST-Segment Elevation. Circulation, 100, 1660-1666. https://doi.org/10.1161/01.CIR.100.15.1660

[21] Coronel, R., and Antzelevitch, C. (2005) Right Ventricular Fibrosis and Conduction Delay in a Patient with Clinical Signs of Brugada Syndrome: A Combined Electrophysiological, Genetic, Histopathologic, and Computational Study. Circulation, 112, 2769-2777. https://doi.org/10.1161/CIRCULATIONAHA.105.532614 
[22] Hoogendijk, M.G., et al. (2010) The Brugada ECG Pattern: A Marker of Channelopathy, Structural Heart Disease, or Neither? Toward a Unifying Mechanism of the Brugada Syndrome. Circulation: Arrhythmia and Electrophysiology, 3, 283-290. https://doi.org/10.1161/CIRCEP.110.937029

[23] Meregalli, P.G., Wilde, A.A. and Tan, H.L. (2005) Pathophysiological Mechanisms of Brugada Syndrome: Depolarization Disorder, Repolarization Disorder, or More? Cardiovascular Research, 67, 367-378. https://doi.org/10.1016/j.cardiores.2005.03.005

[24] Wilde, A.A., et al. (2010) The Pathophysiological Mechanism Underlying Brugada Syndrome: Depolarization versus Repolarization. Journal of Molecular and Cellular Cardiology, 49, 543-553. https://doi.org/10.1016/j.yjmcc.2010.07.012

[25] Antzelevitch, C. (2006) Brugada Syndrome. Pacing and Clinical Electrophysiology, 29, 1130-1159. https://doi.org/10.1111/j.1540-8159.2006.00507.x

[26] Lukas, A. and Antzelevitch, C. (1996) Phase 2 Reentry as a Mechanism of Initiation of Circus Movement Reentry in Canine Epicardium Exposed to Simulated Ischemia. Cardiovascular Research, 32, 593-603. https://doi.org/10.1016/S0008-6363(96)00115-0

[27] Tukkie, R., Sogaard, P., Vleugels, J., de Groot, I.K.L.M., Wilde, A.A.M. and Tan, H.L. (2004) Delay in Right Ventricular Activation Contributes to Brugada Syndrome. Circulation, 109, 1272-1277 https://doi.org/10.1161/01.CIR.0000118467.53182.D1

[28] Perez-Riera, A.R., et al. (2012) Do Patients with Electrocardiographic Brugada Type 1 Pattern Have Associated Right Bundle Branch Block? A Comparative Vectorcardiographic Study. Europace, 14, 889-897. https://doi.org/10.1093/europace/eur395

[29] Corrado, D., et al. (1996) Familial Cardiomyopathy Underlies Syndrome of Right Bundle Branch Block, ST Segment Elevation and Sudden Death. Journal of the American College of Cardiology, 27, 443-448. https://doi.org/10.1016/0735-1097(95)00485-8

[30] Frustaci, A., et al. (2005) Cardiac Histological Substrate in Patients with Clinical Phenotype of Brugada Syndrome. Circulation, 112, 3680-3687. https://doi.org/10.1161/CIRCULATIONAHA.105.520999

[31] Tada, H., et al. (1998) Arrhythmogenic Right Ventricular Cardiomyopathy Underlies Syndrome of Right Bundle Branch Block, ST-Segment Elevation, and Sudden Death. American Journal of Cardiology, 81, 519-522. https://doi.org/10.1016/S0002-9149(97)00942-9

[32] Furushima, H., et al. (2007) Comparison of Conduction Delay in the Right Ventricular Outflow Tract between Brugada Syndrome and Right Ventricular Cardiomyopathy: Investigation of Signal Average ECG in the Precordial Leads. Europace, 9, 951-956. https://doi.org/10.1093/europace/eum128

[33] Takagi, M., Kuribayashi, S., Taguchi, A., Kurita, T., Suyama, K., Kamakura, S. and Takamiya, M. (2003) Abnormal Response to Sodium Channel Blockers in Patients with Brugada Syndrome: Augmented Localised Wall Motion Abnormalities in the Right Ventricular Outflow Tract Region Detected by Electron Beam Computed Tomography. Heart, 89, 169-174. https://doi.org/10.1136/heart.89.2.169

[34] Capulzini, L., Brugada, P., Brugada, J. and Brugada, R. (2010) Arrhythmia and Right Heart Disease: From Genetic Basis to Clinical Practice. Revista Española de Cardiología, 63, 963-983. https://doi.org/10.1016/S0300-8932(10)70208-6

[35] Priori, S.G., et al. (2002) Natural History of Brugada Syndrome: Insights for Risk Stratification and Management. Circulation, 105, 1342-1347. 
https://doi.org/10.1161/hc1102.105288

[36] Kusano, K.F., et al. (2008) Atrial Fibrillation in Patients with Brugada Syndrome Relationships of Gene Mutation, Electrophysiology, and Clinical Backgrounds. Journal of the American College of Cardiology, 51, 1169-1175. https://doi.org/10.1016/j.jacc.2007.10.060

[37] Schimpf, R., et al. (2008) Prevalence of Supraventricular Tachyarrhythmias in a Cohort of 115 Patients with Brugada Syndrome. Annals of Noninvasive Electrocardiology, 13, 266-269. https://doi.org/10.1111/j.1542-474X.2008.00230.x

[38] Eckardt, L., Kirchhof, P., Johna, R., Haverkamp, W., Breithardt, G. and Borggrefe, M. (2001) Wolff-Parkinson-White Syndrome Associated with Brugada Syndrome. Pacing and Clinical Electrophysiology, 24, 1423-1424. https://doi.org/10.1046/j.1460-9592.2001.01423.x

[39] Letsas, K.P., et al. (2013) Sinus Node Disease in Subjects with Type 1 ECG Pattern of Brugada Syndrome. Journal of Cardiology, 61, 227-231. https://doi.org/10.1016/j.jjcc.2012.12.006

[40] Mizumaki, K., et al. (2004) Vagal Activity Modulates Spontaneous Augmentation of ST Elevation in the Daily Life of Patients with Brugada Syndrome. Journal of Cardiovascular Electrophysiology, 15, 667-673. https://doi.org/10.1046/j.1540-8167.2004.03601.x

[41] Takigawa, M., et al. (2008) Seasonal and Circadian Distributions of Ventricular Fibrillation in Patients with Brugada Syndrome. Heart Rhythm, 5, 1523-1527. https://doi.org/10.1016/j.hrthm.2008.08.022

[42] Chockalingam, P., et al. (2011) Fever-Induced Life-Threatening Arrhythmias in Children Harboring an SCN5A Mutation. Pediatrics, 127, e239-e244. https://doi.org/10.1542/peds.2010-1688

[43] Litovsky, S.H. and Antzelevitch, C. (1990) Differences in the Electrophysiological Response of Canine Ventricular Subendocardium and Subepicardium to Acetylcholine and Isoproterenol. A Direct Effect of Acetylcholine in Ventricular Myocardium. Circulation Research, 67, 615-627. https://doi.org/10.1161/01.RES.67.3.615

[44] Napolitano, C. and Priori, S.G. (2006) Brugada Syndrome. Orphanet Journal of Rare Diseases, 1, 35. https://doi.org/10.1186/1750-1172-1-35

[45] Morita, H., et al. (2008) Fragmented QRS as a Marker of Conduction Abnormality and a Predictor of Prognosis of Brugada Syndrome. Circulation, 118, 1697-1704. https://doi.org/10.1161/CIRCULATIONAHA.108.770917

[46] Pitzalis, M.V., Anaclerio, A., Iacoviello, M., et al. (2003) QT-Interval Prolongation in Right Precordial Leads: An Additional Electrocardiographic Hallmark of Brugada Syndrome. Journal of the American College of Cardiology, 42, 1632-1637. https://doi.org/10.1016/j.jacc.2003.07.005

[47] Brugada, J., Brugada, R., Antzelevitch, C., et al. (2002) Long-Term Follow-Up of Individuals with the Electrocardiographic Pattern of Right Bundle-Branch Block and ST-Segment Elevation in Precordial Leads V1 to V3. Circulation, 105, 73-78. https://doi.org/10.1161/hc0102.101354

[48] Brugada, J., Brugada, R. and Brugada, P. (2003) Determinants of Sudden Cardiac Death in Individuals with the Electrocardiographic Pattern of Brugada Syndrome and No Previous Cardiac arrest. Circulation, 108, 3092-3096. https://doi.org/10.1161/01.CIR.0000104568.13957.4F

[49] Eckardt, L., Probst, V., Smits, J.P.P., et al. (2005) Long-Term Prognosis of Individuals with Right Precordial ST-Segment-Elevation Brugada Syndrome. Circulation, 
111, 257-263. https://doi.org/10.1161/01.CIR.0000153267.21278.8D

[50] Di Diego, J.M., Cordeiro, J.M., Goodrow, R.J., et al. (2002) Ionic and Cellular Basis for the Predominance of the Brugada Syndrome Phenotype in Males. Circulation, 106, 2004-2011. https://doi.org/10.1161/01.CIR.0000032002.22105.7A

[51] Matsuo, K., Akahoshi, M., Seto, S. and Yano, K. (2003) Disappearance of the Brugada-Type Electrocardiogram after Surgical Castration: A Role for Testosterone and an Explanation for the Male Preponderance. Pacing and Clinical Electrophysiology, 26, 1551-1553. https://doi.org/10.1046/j.1460-9592.2003.t01-1-00227.x

[52] Shimizu, W., Matsuo, K., Kokubo, Y., et al. (2007) Sex Hormone and Gender Difference-Role of Testosterone on Male Predominance in Brugada Syndrome. Journal of Cardiovascular Electrophysiology, 18, 415-421. https://doi.org/10.1111/j.1540-8167.2006.00743.x

[53] Juang, J.J. and Horie, M. (2016) Genetics of Brugada Syndrome. Journal of Arrhythmia, 32, 418-425. https://doi.org/10.1016/j.joa.2016.07.012

[54] Benito, B., Sarkozy, A., Mont, L., et al. (2008) Gender Differences in Clinical Manifestations of Brugada Syndrome. Journal of the American College of Cardiology, 52, 1567-1573. https://doi.org/10.1016/j.jacc.2008.07.052

[55] Probst, V., Veltmann, C., Eckardt, L., et al. (2010) Long-Term Prognosis of Patients Diagnosed with Brugada Syndrome: Results from the FINGER Brugada Syndrome Registry. Circulation, 121, 635-643. https://doi.org/10.1161/CIRCULATIONAHA.109.887026

[56] Wilde, A.A., Antzelevitch, C., Borggrefe, M., et al. (2002) Proposed Diagnostic Criteria for the Brugada Syndrome: Consensus Report. Circulation, 106, 2514-2519. https://doi.org/10.1161/01.CIR.0000034169.45752.4A

[57] Priori, S.G., Wilde, A.A., Horie, M., Cho, Y., et al. (2013) Executive Summary: HRS/EHRA/APHRS Expert Consensus Statement on the Diagnosis and Management of Patients with Inherited Primary Arrhythmia Syndromes. Europace, 15, 1389-1406. https://doi.org/10.1093/europace/eut272

[58] Priori, S.G., Wilde, A.A., Horie, M., et al. (2013) HRS/EHRA/APHRS Expert Consensus Statement on the Diagnosis and Management of Patients with Inherited Primary Arrhythmia Syndromes: Document Endorsed by HRS, EHRA, and APHRS in May 2013 and by ACCF, AHA, PACES, and AEPC in June 2013. Heart Rhythm, 10, 1932-1963. https://doi.org/10.1016/j.hrthm.2013.05.014

[59] Yap, Y.G., Behr, E.R. and Camm, A.J. (2009) Drug-Induced Brugada Syndrome. Europace, 11, 989-994. https://doi.org/10.1093/europace/eup114

[60] Bayes de Luna, A., Brugada, J., Baranchuk, A., et al. (2012) Current Electrocardiographic Criteria for Diagnosis of Brugada Pattern: A Consensus Report. Journal of Electrocardiology, 45, 433-442. https://doi.org/10.1016/j.jelectrocard.2012.06.004

[61] Sangwatanaroj, S., Prechawat, S., Sunsaneewitayakul, B., Sitthisook, S., Tosukhowong, P. and Tungsanga, K. (2001) New Electrocardiographic Leads and the Procainamide Test for the Detection of the Brugada Sign in Sudden Unexplained Death Syndrome Survivors and Their Relatives. European Heart Journal, 22, 2290-2296. https://doi.org/10.1053/euhj.2001.2691

[62] Hong, K., Brugada, J., Oliva, A., Berruezo-Sanchez, A., Potenza, D., et al. (2004) Value of Electrocardiographic Parameters and Ajmaline Test in the Diagnosis of Brugada Syndrome Caused by SCN5A Mutations. Circulation, 110, 3023-3027. https://doi.org/10.1161/01.CIR.0000144299.17008.07

[63] Dumaine, R., Towbin, J.A., Brugada, P., Vatta, M., Nesterenko, D.V., et al. (1999) 
Ionic Mechanisms Responsible for the Electrocardiographic Phenotype of the Brugada Syndrome Are Temperature Dependent. Circulation Research, 85, 803-809. https://doi.org/10.1161/01.RES.85.9.803

[64] Porres, J.M., Brugada, J., Urbistondo, V., GarcÍA, F., Reviejo, K. and Marco, P. (2002) Fever Unmasking the Brugada Syndrome. Pacing and Clinical Electrophysiology, 25, 1646-1648. https://doi.org/10.1046/j.1460-9592.2002.01646.x

[65] Ortega-Carnicer, J., Benezet, J. and Ceres, F. (2003) Fever-Induced ST-Segment Elevation and T-Wave Alternans in a Patient with Brugada Syndrome. Resuscitation, 57, 315-317. https://doi.org/10.1016/S0300-9572(03)00057-1

[66] De Bliek, E.C. (2018) ST Elevation: Differential Diagnosis and Caveats. A Comprehensive Review to Help Distinguish ST Elevation Myocardial Infarction from Nonischemic Etiologies of ST Elevation. Turkish Journal of Emergency Medicine, 18, 1-10. https://doi.org/10.1016/j.tjem.2018.01.008

[67] Baum, B., Ingaramo, O. and Chao, D. (2018) Adolescent Seizure in the Emergency Department Due to Concomitant Brugada Syndrome. Pediatric Emergency Care, 34, e144-e146. https://doi.org/10.1097/PEC.0000000000001508

[68] Postema, P.G., Wolpert, C., Amin, A.S., Probst, V., Borggrefe, M., et al. (2009) Drugs and Brugada Syndrome Patients: Review of the Literature, Recommendations, and an Up-to-Date Website. Heart Rhythm, 6, 1335-1341.

http://www.brugadadrugs.org https://doi.org/10.1016/j.hrthm.2009.07.002

[69] Duque, M., et al. (2017) Anesthesia and Brugada Syndrome: A 12-Year Case Series. Journal of Clinical Anesthesia, 36, 168-173. https://doi.org/10.1016/j.jclinane.2016.09.031

[70] Belhassen, B., Glick, A. and Viskin, S. (2004) Efficacy of Quinidine in High-Risk Patients with Brugada Syndrome. Circulation, 110, 1731-1737. https://doi.org/10.1161/01.CIR.0000143159.30585.90

[71] Schweizer, P.A., Becker, R., Katus, H.A. and Thomas, D. (2010) Successful Acute and Long-Term Management of Electrical Storm in Brugada Syndrome Using Orciprenaline and Quinine/Quinidine. Clinical Research in Cardiology, 99, 467-470. https://doi.org/10.1007/s00392-010-0145-7

[72] Hermida, J.S., Denjoy, I., Clerc, J., Extramiana, F., Jarr, G.M, et al. (2004) Hydroquinidine Therapy in Brugada Syndrome. Journal of the American College of Cardiology, 43, 1853-1860. https://doi.org/10.1016/j.jacc.2003.12.046

[73] Marquez, M.F., Bonny, A., Hernández-Castillo, E., De Sisti, A., Gómez-Flores, J., et al. (2012) Long-Term Efficacy of Low Doses of Quinidine on Malignant Arrhythmias in Brugada Syndrome with an Implantable Cardioverter-Defibrillator: A Case Series and Literature Review. Heart Rhythm, 9, 1995-2000. https://doi.org/10.1016/j.hrthm.2012.08.027

[74] Probst, V., Denjoy, I., Meregalli, P.G., Amirault, G.-C., et al. (2007) Clinical Aspects and Prognosis of Brugada Syndrome in Children. Circulation, 115, 2042-2048. https://doi.org/10.1161/CIRCULATIONAHA.106.664219

[75] Haissaguerre, M., Extramiana, F., Hocini, M., Cauchemez, B., Jaïs, P., et al. (2003) Mapping and Ablation of Ventricular Fibrillation Associated with Long-QT and Brugada Syndromes. Circulation, 108, 925-928. https://doi.org/10.1161/01.CIR.0000088781.99943.95

[76] Nademanee, K., et al. (2011) Prevention of Ventricular Fibrillation Episodes in Brugada Syndrome by Catheter Ablation over the Anterior Right Ventricular Outflow Tract Epicardium. Circulation, 123, 1270-1279. https://doi.org/10.1161/CIRCULATIONAHA.110.972612 


\section{Abbreviations}

ECG: Electrocardiogram.

SCD: Sudden Cardiac Death.

BS: Brugada Syndrome.

PVT: Polymorphic Ventricular Tachycardia.

VF: Ventricular Fibrillations. 